WHITE PAPER FOR THE

PLANETARY SCIENCE DECADAL SURVEY, 2023 - 2032

\title{
Aerocapture as an Enhancing Option for Ice Giants Missions
}

\author{
Primary Author: Soumyo Dutta \\ NASA Langley Research Center \\ Phone: 757-864-3894 \\ E-Mail: soumyo.dutta@nasa.gov
}

\section{Co-Authors:}

Gonçalo Afonso ${ }^{1}$

Samuel W. Albert ${ }^{2}$

Hisham K. Ali ${ }^{3}$

Gary A. Allen ${ }^{4}$

Antonella I. Alunni ${ }^{5}$

James O. Arnold ${ }^{4}$

Alex Austin 6

Gilles Bailet $^{7}$

Shyam Bhaskaran ${ }^{6}$

Alan M. Cassell ${ }^{5}$

George T. Chen ${ }^{6}$

Ian J. Cohen ${ }^{8}$

James A. Cutts ${ }^{6}$

Rohan G. Deshmukh ${ }^{4}$

Robert A. Dillman ${ }^{9}$

Guillermo Dominguez

Calabuig ${ }^{10}$

Sarah N. D'Souza ${ }^{5}$

Donald T. Ellerby ${ }^{5}$

Giusy Falcone ${ }^{11}$

Alberto Fedele ${ }^{12}$

1 Spin.Works S.A.

2 University of Colorado, Boulder

3 Georgia Institute of Technology

4 Analytical Mechanics Associates

5 NASA Ames Research Center

6 Jet Propulsion Laboratory/California Institute of Technology

7 University of Glasgow

8 John Hopkins University/Applied Physics Laboratory

9 NASA Langley Research Center

${ }^{10}$ Deimos Space (Spain)

${ }^{11}$ University of Illinois, Urbana-Champaign

${ }^{12}$ C.I.R.A. Italian Aerospace Research Centre

${ }^{13}$ Purdue University

Ye Lu

\begin{tabular}{|c|c|c|}
\hline Jay Feld & & Zachary R. Putnam ${ }^{11}$ \\
\hline Roberto & & Jeremy R. Rea ${ }^{15}$ \\
\hline Athul P. & & Sachin Alexander Reddy ${ }^{2}$ \\
\hline Tiago $\mathrm{H}$ & & Thomas Reimer ${ }^{22}$ \\
\hline Jeffrey $\mathrm{P}$ & & Sarag J. Saikia ${ }^{23}$ \\
\hline Shayna I & & Isil Sakraker Özmen ${ }^{22}$ \\
\hline Christop & $\operatorname{ian}^{14}$ & Kunio Sayanagi ${ }^{24}$ \\
\hline Vandana & & Stephan Schuster ${ }^{25}$ \\
\hline Breanna & $\mathrm{on}^{15}$ & Jennifer Scully ${ }^{6}$ \\
\hline Craig A. & & Ronald R. Sostaric ${ }^{15}$ \\
\hline Jean-Pie & $\operatorname{ton}^{17}$ & Christophe Sotin 6 \\
\hline Marcus & & David A. Spencer ${ }^{6}$ \\
\hline Ping $\mathrm{Lu}^{1}$ & & Benjamin M. Tackett ${ }^{4}$ \\
\hline Ye Lu ${ }^{19}$ & & Nikolas Trawny ${ }^{6}$ \\
\hline Rafael A & & Ethiraj Venkatapathy ${ }^{5}$ \\
\hline Daniel A & & Paul F. Wercinski ${ }^{5}$ \\
\hline Robert V & & Michael C. Wilder ${ }^{5}$ \\
\hline Michelle & $\mathrm{k}^{9}$ & Michael J. Wright ${ }^{5}$ \\
\hline Adam P. & & Cindy L. Young 9 \\
\hline Miguel I & úcar ${ }^{20}$ & \\
\hline Richard & & \\
\hline & ${ }^{14}$ Univ & fornia, Los Angeles \\
\hline & ${ }^{15} \mathrm{NAS}$ & pace Center \\
\hline & ${ }^{16}$ Univ & souri \\
\hline & ${ }^{17}$ Frenc & Centre for Scientific Research \\
\hline & ${ }^{18}$ San I & Jniversity \\
\hline stitute of & ${ }^{19}$ Kent & sity \\
\hline & ${ }^{20}$ Auro & y B.V. \\
\hline & ${ }^{21}$ Mull & ience Laboratory, University \\
\hline ics & Colle & \\
\hline & ${ }^{22}$ Instit & ures and Design - German \\
\hline & Aero & (DLR) \\
\hline g & $\begin{array}{l}{ }^{24} \mathrm{Spac} \\
{ }^{24} \mathrm{Ham}\end{array}$ & $\begin{array}{l}\text { nologies Inc. } \\
\text { ity }\end{array}$ \\
\hline ntre & ${ }^{25}$ Euro & Agency - ESTEC \\
\hline
\end{tabular}

Jay Feldman ${ }^{5}$

Athul P. Girija ${ }^{13}$

Tiago Hormigo

Jeffrey P. Hill

Christopher Jelloian ${ }^{14}$

(ana Jha

Craig A. Kluever ${ }^{16}$

Marcus A. Lobbia

Ping Lu

Daniel A. Matz ${ }^{15}$

Robert W. Moses ${ }^{9}$

Adam P. Nelessen

Miguel Pérez-Ayúcar ${ }^{20}$

${ }^{14}$ University of California, Los Angeles

${ }^{8}$ San Diego State University

College London

Institute of Structures and Design - German

${ }^{24}$ Hampton University

European Space Agency - ESTEC 


\section{Executive Summary}

Investigation of Uranus and Neptune, via orbiter and atmospheric probes, is required to answer pressing science questions that have been raised in previous Decadal Surveys. As the Ice Giants are the farthest planets from Earth, traditional fully-propulsive orbit insertion missions have transit times to the planetary bodies nearing 13-15 years and require a large amount of propellant, leaving less mass for the scientific payload. Aerocapture uses aerodynamic forces generated by flight within a planetary atmosphere to decelerate and achieve orbit insertion. Although, aerocapture has not been used in the past, recent developments in thermal protection systems, guidance and control, and navigation capabilities enable the use of rigid, heritage entry vehicle configurations already flown at other planetary bodies for Ice Giants aerocapture. With the addition of these recent capabilities, aerocapture can robustly deliver spacecraft to Ice Giant orbits, while substantially increasing on-orbit payload mass (more than 40\%) and reducing the transit time by $2-5$ years (15$30 \%$ ) relative to fully-propulsive orbit insertion.

\section{Motivation for Uranus and Neptune Missions}

Uranus and Neptune, the Ice Giant planets, are continuously identified as high-priority science targets, including in the last Decadal Survey. To date, they have only been visited by Voyager 2 during its flyby in the 1980s. Much of our knowledge about the planets come from these brief encounters. Understanding how the Ice Giants formed and evolved is key to unlocking the history of our solar system and comprehending the mechanisms behind planetary formation. Current planetary formation models show a low probability that Uranus and Neptune should exist, yet many of the discovered exoplanets are a similar size to the Ice Giants. In addition to the planets themselves, their systems (i.e. moons, rings, and magnetosphere) provide an exceptional opportunity for new discoveries. Neptune's moon Triton, in particular, is thought to be a captured Kuiper Belt object and may harbor an active subsurface ocean that could contain extant life.

Given all that needs to be understood at the Ice Giants, a robust and comprehensive science payload is needed, which is fulfilled by having both orbiter and probe elements. Previous studies have indicated the need for an orbiter with a narrow-and wide-angle camera, imaging spectrometer, magnetometer, mass spectrometer, and detectors for plasma waves, plasma and energetic particles, and dust. In addition, an in-situ atmospheric probe is needed to sample the atmosphere and understand the chemical composition. While past studies have released the probe on approach to decrease spacecraft mass and thus the amount of propellant required, it is preferable that the probe is carried to orbit, so that the science team can utilize on-orbit observations and choose a probe target location to maximize science return.

Ice Giants missions are difficult due to the remoteness of their destinations from Earth $(\sim 20$ $\mathrm{AU}$ for Uranus and $\sim 30 \mathrm{AU}$ for Neptune), and the large orbit insertion $\Delta \mathrm{V}$ requirement for trajectories that reach the planets in a reasonable amount of time ( $<13$ years). This in turn results in propulsion system and propellant masses that severely limit available science payload mass and scientific value of the missions. If atmospheric forces reduce the propulsive needs, more mass can be allocated to the science instrumentation and an atmospheric probe, both of which are critical for the mission. Therefore, decreasing the required propellant mass of Ice Giant mission designs can greatly enhance the science value for Uranus and Neptune missions. 


\section{Aerocapture as an Enhancing Technology for Ice Giants Missions}

Aerocapture is an orbital insertion technique which utilizes a single pass through the planetary atmosphere to dissipate enough orbital energy for planetary capture. Small propulsive maneuvers after the aerocapture maneuver correct any insertion errors. Aerocapture is an enhancing technology for science mission at Uranus and Neptune. It can increase the science payload delivered to orbit, can reduce interplanetary transit time, and should provide a cost benefit.

Aerocapture for the Ice Giants has been studied at length. Early studies proposed atmospheric techniques for orbital transfers at different planetary bodies [1-3]. These studies laid the foundation in the scientific community of aerocapture as an orbit insertion technique. At Neptune, large inflated drag surfaces for aerocapture were investigated [4-5]. NASA conducted systems analysis for aerocapture design reference missions at Venus [6], Mars [7], Titan [8], and Neptune [9]. In particular, the Neptune study investigated orbit insertion into a highly elliptical retrograde orbit to enable orbiter flyby of Triton, while deploying two atmospheric probes prior to aerocapture However, in order to achieve the control authority to conduct aerocapture given the limited atmospheric knowledge and higher approach navigation uncertainty, a new, high lift design for the entry vehicle was selected. The study demonstrated the feasibility and robustness of aerocapture, but a drawback was the design and qualification of a new entry vehicle configuration.

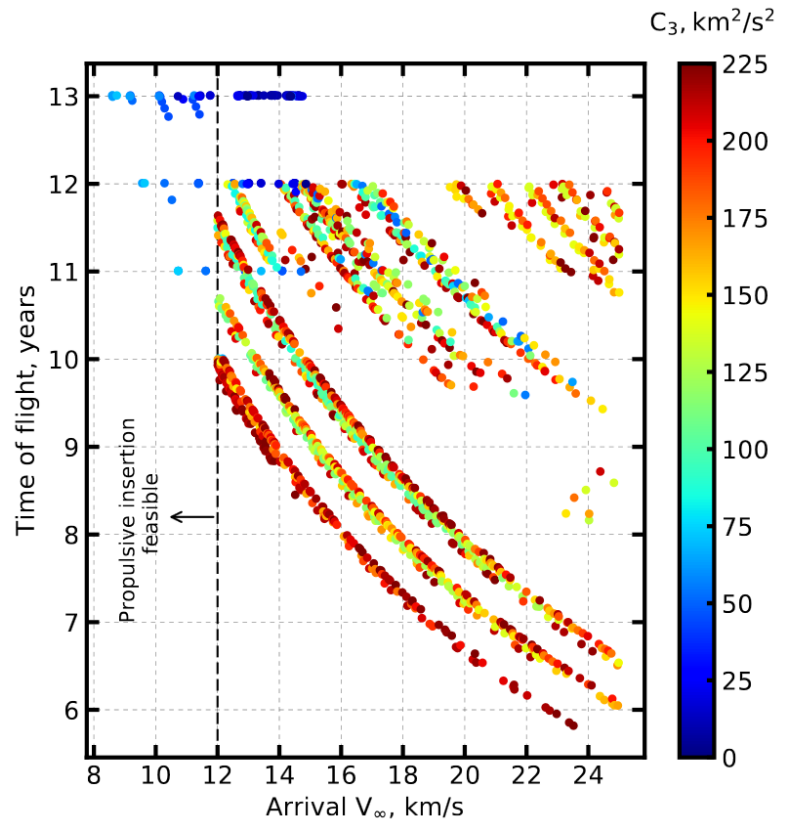

Figure 1. Interplanetary trajectory space to Neptune in 2030s-2040s. [13]

Historically, fully-propulsive options have been used for planetary orbiters. Studies have shown that for the same launch vehicle, aerocapture can deliver more mass to orbit than a fully-propulsive orbit insertion design $[9,10]$. Aerocapture allows for flexibility in the necessary arrival conditions at Uranus and Neptune as the interplanetary trajectory could be faster than traditional designs since the aerocapture maneuver can attain a large $\Delta \mathrm{V}$ during the atmospheric segment [11]. This in turn allows for shorter transit times and for science data return to occur earlier [12]. For these short transit time trajectories, the fully-propulsive design may require an increased propellant mass that eliminates mass dedicated to science payload or require a higher performance launch vehicle. Some of the benefits of aerocapture over fullypropulsive orbit insertion maneuvers (Fig. 1) include transit times less than 10 years which are not feasible without aerocapture [13]. Aerocapture can allow for reduced launch costs without significant compromise to the delivered science payload. Moreover, in situ atmospheric data can be captured during the atmospheric flight portion of aerocapture, yielding valuable science data.

Studies looking at aerocapture for Ice Giants have consistently shown to deliver more onorbit science payload (more than 40\%) over traditional fully-propulsive planetary insertion maneuvers and the interplanetary trajectories are 2 to 5 years shorter $[9,13]$. However, when some of these studies were considered 15 years ago, the aerocapture technologies, especially in the realm of thermal protection system (TPS), guidance, control, and navigation meant a new class of entry 
vehicle would have to be developed to meet the goals for Ice Giants missions. Recent technological developments show that aerocapture at the Ice Giants may not require a new class of entry vehicles. In fact, these new capabilities make heritage entry vehicle configurations flown at Earth, Mars, Venus, Titan, and Jupiter feasible for conducting Ice Giants aerocapture with robust performance.

\section{New Capabilities Make Aerocapture Feasible with Heritage Entry Configurations}

Capability 1. New TPS materials have been developed which meet requirements for Uranus and Neptune aerocapture

Aerocapture requires an integrated TPS to protect the spacecraft from thermal and mechanical loads generated during entry. Mass efficient and robust TPS is needed as any amount allocated to TPS reduces the allocation for the payload, while any TPS failure will result in a loss of mission. Ice Giants missions, due to the large $\Delta \mathrm{V}$ required for orbit insertion, require a combination of ablative and insulative TPS that can endure high heat rate, stagnation pressure, and heat load for mass efficient design. Heatshield for Extreme Entry Environment Technology (HEEET) is a new mass efficient and robust TPS that is capable of supporting Ice Giants Missions [14]. HEEET was developed with Ice Giants probe missions in mind, as heritage carbon phenolic used for missions like Jupiter Galileo is no longer available and would not be as mass efficient as HEEET. HEEET has been matured to Technology Readiness Level (TRL) 6, needed for a number of destinations, and a variant of HEEET is the baseline for the Mars Sample Return (MSR) Earth Entry Vehicle (EEV). A companion white paper [15] provides recommendations about why and how HEEET and Phenolic Impregnated Carbon Ablator (PICA) will be sustained by NASA, as these TPS materials enable various planetary in-situ missions in the coming decades. Ref [16] discusses recent TPS development specifically geared for the Outer Planets.

In support for this paper, a TPS sizing study with HEEET was conducted with a range of potential aerocapture configurations for the Ice Giant planets. Ref [16] contains the detailed results of the exercise, in which vehicles with ballistic coefficients in the range of $75-350 \mathrm{~kg} / \mathrm{m}^{2}$ (a large variety that encompasses many potential Ice Giant orbiter and probe configurations) and a range of potential arrival velocities to the Ice Giants for the 2030-2040 time period were considered. The approach taken was to perform bounding analyses, where the aerocapture vehicle was flown with the lift force vector up or lift force vector down within the atmosphere to capture potential variations in heat flux, heat load, peak sensed acceleration, and peak stagnation pressure. These conditions provided guidance for the choice of TPS materials. Peak stagnation heat flux of the order 200 to $3300 \mathrm{~W} / \mathrm{cm}^{2}$ and peak stagnation pressure between 0.015 to $1.25 \mathrm{~atm}$ were observed. Although PICA could be used for some of the lower arrival velocity conditions, the only TPS capable for the full range is HEEET in its insulting and dual layer variants. A conclusion from the study is that HEEET is very capable of meeting the broad range of conditions. For typical ballistic coefficients expected of aerocapture missions to the Ice Giants $\left(150 \mathrm{~kg} / \mathrm{m}^{2}\right)$, the fully margined TPS mass fraction will be of the order of 20 to $40 \%$ with an insulating layer of HEEET for TPS.

\section{Capability 2. Guidance and control schemes have been developed that enable aerocapture under robust conditions with existing entry vehicle configuration}

Past studies that have considered aerocapture for the Ice Giants have noted the need for higher control authority for robust aerocapture performance $[9,17]$. These studies have usually shown the need for a newer class of entry vehicle that has higher lift-to-drag ratios, but these vehicles are conceptual and the hardware does not exist. Some of the reasons for the need for a larger control authority have been atmospheric knowledge uncertainty for the Ice Giants planets and the 
uncertainty of the arrival conditions at atmospheric entry due to interplanetary navigation, the ephemeris of the planets, and the large distances from Earth, which are topics discussed in the next section. However, recent work with modern guidance and control schemes have shown that robust aerocapture can be performed at the Ice Giants with lower control authority provided by heritage entry vehicle configurations, even with large atmospheric uncertainties.

Uncertainty in atmospheric conditions is still a key sensitivity for aerocapture guidance schemes that command the spacecraft during the atmospheric pass. However, the sensitivity can be lessened by using predictor-corrector algorithms that can adapt with the knowledge of the atmosphere during the inbound portion of the aerocapture maneuver. In the past, Ice Giants aerocapture studies had considered analytical algorithms that were tuned pre-flight to target exit conditions and had an assumption of the atmospheric profile built-in to the parameters. Large control authority was needed by the spacecraft to adjust the trajectory when atmospheric and ensuing aerodynamic conditions were different from the reference trajectory. Predictor-corrector algorithms, which are proposed for human-scale flights like Orion, continue adjusting their commanded profiles throughout the atmospheric flight and can adapt when conditions are different from pre-generated reference profiles. Although there is higher computation complexity associated with these algorithms, recent studies have shown that in similar situations, predictor-corrector schemes accomplish the aerocapture maneuver with less correction burn fuel $[18,19]$.

Moreover, past studies had focused on a control mechanism known as bank angle modulation (BAM), where the vehicle rotates around the velocity vector to point the trimmed aerodynamic lift force. The BAM method has been applied previously in planetary missions but rotating the lift force vector alone may not offer enough control authority during aerocapture in uncertain atmospheres. However, since the past studies from Ref. [9], development has occurred in other control mechanisms such as drag modulation (DM) and direct force control (DFC). In $\mathrm{DM}$, instead of controlling the lift force, the vehicle changes the effective drag force on the vehicle to achieve the necessary $\Delta \mathrm{V}$ during the aerocapture maneuver. Multiple methods exist for achieving the differential drag, including towing an inflatable behind the vehicle [20], having a larger aeroshell that is jettisoned at an opportune time during the maneuver [21], or having a mechanism that can continuously change the aerodynamic shape of the vehicle to generate the required drag force. The DFC method directly controls the vehicle angle-of-attack and hence the lift and drag forces so the spacecraft can target the appropriate force at various stages of aerocapture. Mechanisms that allow for DFC include aerodynamic flaps [22] and center-of-mass movement, which has been demonstrated in Earth-based applications [23].

Recent studies that considered advances in guidance algorithms and control mechanisms show that heritage vehicle configurations with lower lift-to-drag capabilities (lower than 0.4) can still robustly accomplish aerocapture at the Ice Giants, where atmospheric uncertainty is large. Compared to past studies that recommended new vehicle development for aerocapture, the new guidance and control strategies make existing vehicle configurations feasible for aerocapture [13].

\section{Capability 3. New navigation abilities improve vehicle state knowledge for Ice Giant missions}

Navigation to the Ice Giants employs standard radiometric tracking from the Deep Space Network augmented by optical navigation (OpNav), as was done on the Voyager 2 spacecraft in its encounter with Uranus and Neptune. OpNav is a technique that uses an onboard camera to image a planetary body with respect to the background stars, which in turn improves the knowledge of the angular position of the spacecraft with respect to the planetary body. The updated relative 
angular position along with standard radiometric tracking data vastly improves the knowledge of the spacecraft trajectory with respect to a planetary body and is required for outer planet missions where the uncertainty in the planetary body's ephemeris is a dominant error source. Currently, most navigation, including OpNav, is ground-based, requiring many hours to days to turn groundreceived navigation data into commands for maneuvering the spacecraft. This is particularly an issue with Ice Giants where the round-trip communication time is over 8 hours. Using standard ground-based navigation from the Deep Space Network, the knowledge uncertainty of the flight path angle at atmospheric interface is larger than the control capability of existing entry vehicle configurations. Navigation uncertainty is one of the main reasons past Ice Giants aerocapture studies have recommended the development of higher lift-to-drag entry vehicles [9].

However, recent advances in onboard autonomous optical navigation (AutoNav) can substantially improve navigation performance [24]. During AutoNav, the processing of onboard images, filtering the data into an orbit solution, and computing maneuvers all occur on the spacecraft flight computer, introducing the capability to act autonomously on late-breaking navigation information and conduct trajectory corrections. Since the optical data accuracy increases linearly with decreasing distance, updated navigation information in the hours and minutes prior to entry can be used to reduce delivery error and state knowledge uncertainty.

Improved navigational uncertainty by itself lowers the lift-to-drag ratio needed for robust aerocapture performance at the Ice Giants [13]. Even without newer guidance and control schemes, results show successful aerocapture and low $\Delta \mathrm{V}$ requirements with lift-to-drag ratios that are achievable with heritage entry vehicle configuration. With the addition of other guidance and control capabilities discussed earlier, aerocapture with heritage entry vehicle configurations will provide a large mass and transit time savings compared to fully-propulsive alternatives.

\section{Why Is Aerocapture Ready for Ice Giant Missions}

The previous sections discussed the mission-enhancing nature of aerocapture, especially for Ice Giant missions where aerocapture can significantly increase on-orbit mass and decrease transit time. So, it is appropriate to also address perceived challenges of aerocapture that have prevented it from being considered as the baseline reference for past Ice Giants mission proposals.

Although aerocapture risk has long been debated in the planetary science and entry communities, consider the risk in the context of past missions, policies, and organizational cultures. The most relevant historical perspective involves aerocapture at Mars. NASA accepted aerocapture as the baseline mission mode for the proposed Mars 2001 Orbiter mission which matured to Phase B but was then cancelled in the wake of the Mars Polar Lander and Mars Climate Orbiter failures in 1999. Since then, NASA has pursued a more conservative approach, especially for entry, descent and landing (EDL) systems where only incremental changes have been made to the 1970's Viking-heritage EDL technology only when deemed necessary.

Additionally, aerocapture has been shown to have less risk than aerobraking, an atmospheric maneuver where the spacecraft is captured in a highly elliptical orbit with fullypropulsive orbit insertion and then the target orbit is achieved with multiple passes through the atmosphere. Aerobraking is a currently-accepted maneuver used for multiple orbiter insertions since the Mars Global Surveyor mission. NASA accepted aerobraking after demonstration of the maneuver by the Venus Magellan mission after primary mission objectives were completed. However, when aerocapture was compared to aerobraking in a probabilistic risk assessment [25], aerocapture was found to have numerically lower risk and had a comparable risk factor to fully- 
propulsive orbit insertion. Compared to aerobraking's high sensitivity to guidance, navigation, and control errors during deep dip operations, aerocapture's sensitivity to atmospheric uncertainties are smaller as the on-board guidance can adapt during flight and the spacecraft can correct execution errors with small $\Delta \mathrm{V}$ applied after atmospheric exit.

Furthermore, the aerocapture maneuver is less complex than EDL. Aerocapture is contained within the hypersonic flight regime, so that instabilities and staging events that occur in supersonic and subsonic regimes of EDL do not apply. Jettisoning of the heatshield outside the atmosphere is the only staging event and it is similar to launch shroud separation. If an aerocapture maneuver is not executed perfectly, the consequence is a higher or lower exit altitude than desired. The off-nominal altitude can be remedied by carrying contingency propellant, still a tiny fraction compared to what would be required for fully-propulsive insertion or aerobraking.

The uncertainty in aerocapture development comes primarily from two subsystems: TPS and guidance algorithms [26]. With the development of HEEET and PICA, NASA has TPS solutions for most planetary entry and aerocapture needs, and a minimum number of materials to sustain. Therefore, TPS for aerocapture applications would no longer be considered a key technology risk. Similarly, hypersonic guidance has improved. Mars Science Laboratory successfully used hypersonic guidance to precisely target the parachute deployment condition during EDL, a problem with more exacting constraints than planetary aerocapture. Hypersonic guidance and numerical predictor-corrector algorithms have been demonstrated during NASA's Exploration Flight Test 1. Additionally, Artemis-1, scheduled for a Lunar return flight test, will further bolster the confidence in similar guidance approaches. Even without a specific demonstration of aerocapture guidance, the performance of the advanced guidance schemes can be bounded by successful demonstration of similar and more stringent surrogate missions

Aerocapture missions also face the challenge of packaging an orbiter with a large highgain antenna and radioisotope power systems needed for Ice Giant missions within the confines of a closed entry capsule. These concerns have been ameliorated by recent work [27], where a flagship-class Uranus orbiter, with radioisotope power systems, high-gain antenna, and entry probe were packaged within a heritage blunt-body entry configuration.

Aerocapture system will be designed using the same heritage tools and methodologies that have been applied to every successful NASA EDL system since Mars Pathfinder. The models that are incorporated into the system design process and the efficiency of the overall process have improved greatly over the past 20 years. Each uncertainty, environmental or vehicular, is accounted for in a robust way with appropriate margins for uncertainties. All of the features of aerocapture, along with the expertise and technologies that have been developed over NASA's EDL missions in the last 20 years, give high confidence in the ability to successfully implement aerocapture for planetary science destinations, especially the Ice Giants, where aerocapture provides a tremendous advantage in on-orbit mass and transit time. A companion white paper describes the applicability of aerocapture to a wide array of planetary science missions [28].

\section{References}

[1] London, H.S, "Change of Satellite Orbit Plane by Aerodynamic Maneuvering," J. Aero Sciences, 1962.

[2] Walberg, G., “A Survey of Aaeroassisted Orbit Transfer”, J. Spacecr Rockets, 1985.

[3] Repic, E. et al., "Aerobraking as a Potential Planetary Capture Mode", J. Spacecr Rockets, 1968. 
[4] Lyons, D.T, et al., "Ballute Aerocapture Trajectories at Neptune", AIAA 2004-5181.

[5] McRonald, A.D., "A Light-Weight Hypersonic Inflatable Drag Device for a Neptune Orbiter", AAS 00-170.

[6] Lockwood, M.K et al., "Systems Analysis for a Venus Aerocapture Mission”, TM-2006214291

[7] Wright, H.S. et al., "Mars Aerocapture Systems Study”, NASA TM-2006-214522

[8] Lockwood, M.K et al., "Aerocapture Systems Analysis for a Titan Mission”, NASA TM-2006214273

[9] Lockwood, M.K et al., “Aerocapture Systems Analysis for a Neptune Mission”, NASA TM2006-214300

[10] Wercinski, P. et al. "Aerocapture Technology Development Needs for Outer Planet Exploration", NASA TM-2002-211386.

[11] Saikia et al. Aerocapture Assessment at Uranus and Neptune for NASA's Ice Giant Studies, Purdue Univ. 2016-MC-0001.

[12] Noca, M.A., et al., "Mission Trades for Aerocapture at Neptune", AIAA 2004-3843.

[13] Girija, A.P et. al, "Feasibility and Performance Analysis of Neptune Aerocapture using Heritage-Blunt Body Aeroshells" J. Spacecr Rockets, 2020

[14] Venkatapathy, E., et al. "Entry System Technology Readiness for Ice-Giant Probe Missions." Space Science Reviews, 2020.

[15] Venkatapathy, E., et al., "Sustaining Mature Thermal Protection Systems Crucial for Future In-Situ Planetary Missions," Planetary Science Decadal Survey 2023-2032.

[16] Ellerby, D. et al., "Technologies for Future Outer Planet Exploration," Planetary Science Decadal Survey 2023-2032.

[17] Spilker, T. et al. "Qualitative Assessment of Aerocapture and Applications to Future Missions," J Spacecr Rockets, 2019.

[18] Deshmukh, R., et al., "Investigation of Direct Force Control for Aerocapture at Neptune," Acta Astronautica, 2020

[19] Heidrich, C. et. al, "Modern Aerocapture Guidance to Enable Reduced-Lift Vehicles at Neptune," AAS 19-221, 2019.

[20] Westhelle, C.H.. et. al, "Assessment of Aerocapture Flight at Titan Using a Drag-Only Device," AIAA 2003-5389.

[21] Putnam, Z.R. et. al, "Drag-Modulation Flight-Control System Options for Planetary Aerocapture," J Spacecr Rockets, 2014

[22] Fedele, A. et. al, "Aerodynamic Control System for a Deployable Re-Entry Capsule," Acta Astronautica, 2020

[23] Olds, A.D. et. al, "IRVE-3 Post-Flight Reconstruction,", AIAA 2013-1390.

[24] Bhaskaran, S., “Autonomous Navigation for Deep Space Missions”, SpaceOps Conf, 2012.

[25] Percy, T.K. et. al, "Assessing the Relative Risk of Aerocapture Using Probabilistic Risk Assessment," AIAA 2005-4107.

[26] Hall, J.L et. al, "Cost-Benefit Analysis of the Aerocapture Mission Set," J. Spacecr Rockets, 2005

[27] Elliot, J. et al., "Mission Design Prospects for the Ice Giants," Ice Giant Systems Workshop, 2020

[28] Austin, A. et al, "Enabling and Enhancing Science Exploration Across the Solar System: Aerocapture Technology for SmallSat to Flagship Missions," Planetary Science Decadal Survey 2023-2032. 